Highly Energetic Physical Processes and

Mechanisms for Emission from Astrophysical Plasmas

IAU Symposium, Vol. 195, 2000

P. C. H. Martens, S. Tsuruta, and M. A. Weber, eds.

\title{
X-ray Radiation from Flare-heated Coronal Plasma
}

\author{
M. Güdel \\ Paul Scherrer Institut, CH-5232 Villigen PSI, Switzerland
}

\begin{abstract}
We investigate the reaction of a coronal loop in the case of repetitive flares, with a power-law distribution in energy, injected into a rigid magnetic loop. Emission measure distributions and temperaturedependent modulations of the radiation are briefly discussed.
\end{abstract}

\section{Introduction and Methods}

There is increasing evidence that a large number of flares play an important role in solar and stellar coronal heating. A superposition of unresolved events may be the cause for the enhanced, quiescent stellar coronal activity, leading to increased statistical fluctuations in hotter plasma components and to characteristic emission measure distributions (Giampapa et al. 1996; Güdel 1997).

Hydrodynamic simulations can ideally be used to model stochastic flares. Kopp \& Poletto (1993) applied a simplified point model approach, while Cargill (1994) used semi-analytic formulae. The results shown here use a 1-D hydrocode (Boris \& Book 1976) that includes treatment of radiative and conductive losses (including conduction saturation) in a circular, symmetric loop (half-length $L=$ $2 \times 10^{9} \mathrm{~cm}$ and loop-top temperature $\left.T_{\text {top }}=1.5 \times 10^{6} \mathrm{~K}\right)$. The lowest $\sim 4 \times 10^{8} \mathrm{~cm}$ contains dense chromospheric gas at $T=8000 \mathrm{~K}$, with corrections for optical depth effects (Nagai 1980). The simulations start with a loop in hydrostatic equilibrium (Serio et al. 1981; Peres et al. 1982). Time-dependent "flare" energy is deposited around the loop top (Gaussian heating profile). The energy input function is composed of a stochastic series of flares; their number distribution in energy $E$ is a power-law, $\mathrm{d} N / \mathrm{d} E \propto E^{-\alpha}$ for a given $\alpha$. The flare duration $d$ follows roughly $d \propto E^{1 / 2}$ (derived from a sample of solar hard X-ray flares).

\section{Results and Outlook}

The most important parameters for the present flare simulations are $\alpha$ and the flare rate above a given lower energy threshold. Figure 1 shows an example for $\alpha=1.9$. The stochastic heating increases $T$ to a "background" of $5-6 \mathrm{MK}$, while the stronger flares superimpose a modulated time profile. The modulation pattern has been investigated in terms of a modulation depth of radiation from plasma at different temperatures, defined as the ratio between the standard deviation and mean of the luminosity time series, both referring to plasma within a given temperature interval. Observationally, such results can be compared to statistical fluctuations in line emission. The modulation depth obviously 

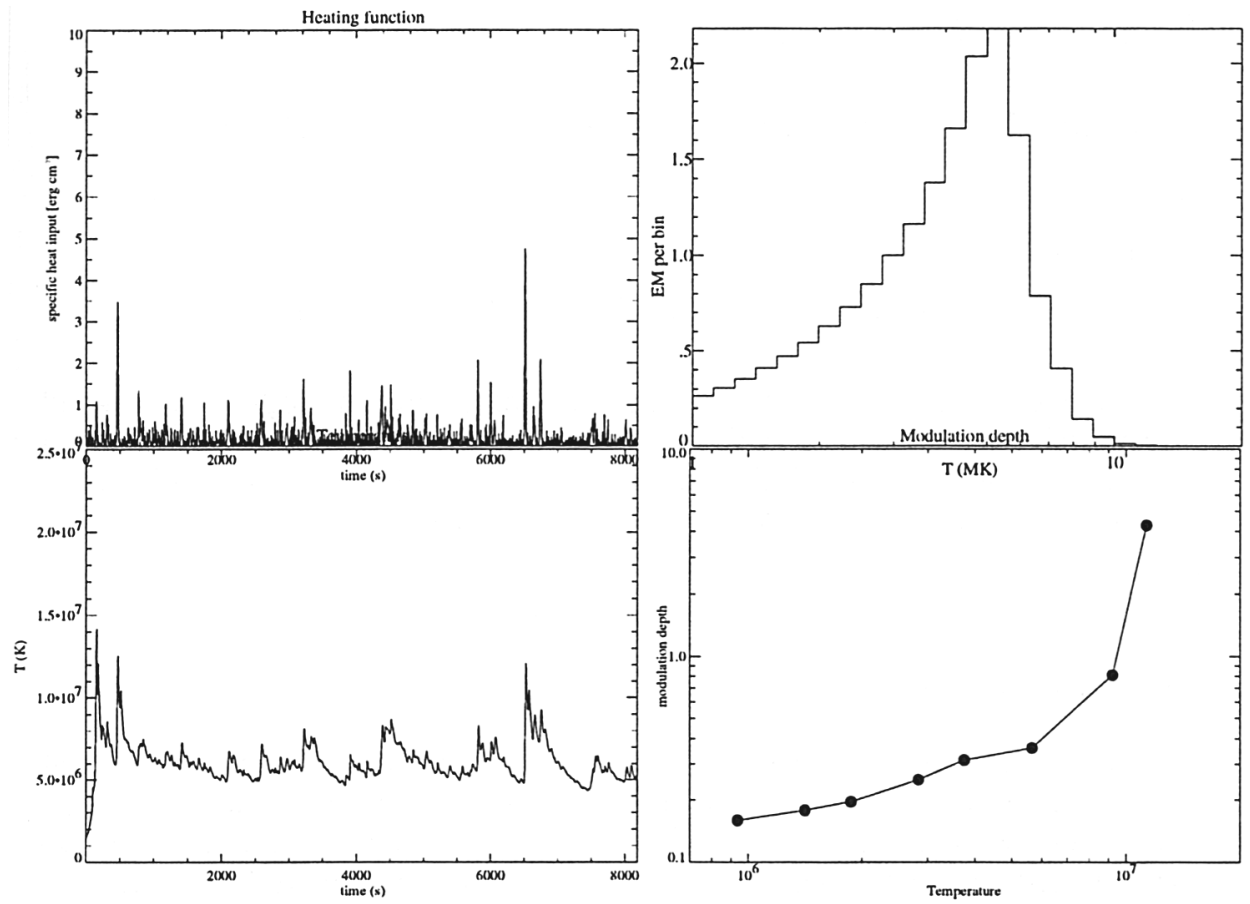

Figure 1. Stochastic flare simulation. Upper left: heat input function. Lower left: reaction of loop top temperature. Upper right: average emission measure distribution. Lower right: modulation depth at different temperatures. Parameters: $\alpha=1.9$, flare rate $0.75 \mathrm{~s}^{-1}$.

increases toward hotter plasma, due to the sparse coverage with sufficiently energetic flares. Giampapa et al. (1996) find that the hotter plasma component in stellar ROSAT observations varies systematically more than the cooler component. Watanabe et al. (1995) interpret rapid fluctuations in the high-T S XV line as evidence for numerous small-scale flare events. Larger samples of simulations will be computed for a systematic study and applications to such observations.

\section{References}

Boris, J. P., \& Book, D. L. 1976, in Meth. Comp. Phys., 16, 85

Cargill, P. J. 1994, ApJ, 422, 381

Giampapa, M. S., Rosner, R., Kashyap, V., Fleming, T. A., Schmitt, J. H. M. M., \& Bookbinder, J. A. 1996, ApJ, 463, 707

Güdel, M. 1997, ApJ, 480, L121

Kopp, R. A., \& Poletto, G. 1993, ApJ, 418, 496

Nagai, F. 1980, Solar Phys., 68, 351

Peres, G., Rosner, R., Serio, S., \& Vaiana, G. S. 1982, ApJ, 252, 791

Serio, S., Peres, G., Vaiana, G. S., Golub, L., \& Rosner, R. 1981, ApJ, 243, 288

Watanabe, T., et al. 1995, Solar Phys., 157, 169 